\title{
The use of membrane technologies in boiler houses of heat and power enterprises
}

\author{
Elena Kuvardina ${ }^{1}$, Natalia Semicheva ${ }^{1}$, Nikolay Kuvardin ${ }^{1}$, Valery Tolmacheva ${ }^{2,}$, Vasily \\ Amelin ${ }^{1}$ \\ ${ }^{1}$ Southwest State University, 94, 50 let Oktyabrya str., 305040, Kursk, Russia \\ ${ }^{2}$ Kursk Institute of education development, 31, Sadovaya str., 305004, Kursk, Russia
}

\begin{abstract}
The article is about the use of membrane technologies in water treatment cycle for boiler houses which use water of superficial reservoirs for operation. Working process of a flat membrane element is described in the article. Movement scheme of purified water streams is introduced. The layer of undesirable deposits which are slowing down membrane operation is formed on membrane surface during its operation. This formed layer is a result of the arising concentration polarization. Impact of low-frequency fluctuations on saturation water stream is offered to decrease concentration polarization which leads to decline in production of membrane devices. Fluctuations were brought in a stream by means of the vibrator. Steady standing waves are in the membrane device as a result of vibration effect. These waves reduce the formation of undesirable deposits layer on a membrane surface. Results received by mathematical modeling and confirmed by experimental studies state that process productivity of ultrafiltration increased by $30 \%$ in frequency range of $60-70 \mathrm{~Hz}$. Frequency range is in standing wave.
\end{abstract}

\section{Introduction}

Boiler-houses' water treatment in various enterprises is an important technological process allowing to ensure effective functioning of boilers. This is a guaranty of their accident-free operation and other thermal equipment of the boiler house during all production cycle in heating season.

Now the majority of boiler houses have material expenditures on water treatment at the expense of annually growth tariffs for water use, deterioration in water quality indicators in sources suitable for industrial use, standards' toughening for quantitative and quality indicators for dumped drains, requirements' increase of conditioned water quality used in technological cycle.[1]

Various artificial water intakes or natural sources such as rivers, ponds are sources of water pumped to the boiler house. The main negative characteristic of these waters is their impurity by various mechanical inclusions, low and high-molecular connections, rigidity salts.

*Corresponding author: 325573@mail.ru 
Impurity of water is changing according to source type. Surface water has more mechanical inclusions, high-molecular connections (HMC), low-molecular connections (LMC) and has less hardness. Hardness of surface water is subjected to noticeable seasonal fluctuations. Maximum value is usually at the end of winter and minimum value is in high water period. In this period it is diluted with soft rain and melt water.

Composition of ground waters is characterized by high content of salts, little content of HMC and LMC and by absolutely low content of mechanical inclusions.

The following shortcomings are noted in water treatment system of heat and power enterprises of Russia: [2]

- extremely rare modernization of water purification filters of boiler houses;

- softening of water for boilers is carried out by ineffective outdated ion-exchange material such as sulphated coal;

- low qualification of staff in boiler houses;

- depreciation of filters in boiler houses.

Different versions of water treatment schemes are used for water preparation of boilers with various powers. The scheme can include the following operations depending on quality of initial water and feed water criteria:

- preliminary water purification (decrease in content of organic substances, suspensions, iron, if necessary reagent softening),

- sodium cation exchange, hydrogen cation exchange with "hungry regeneration", parallel and consecutive hydrogen-sodium-cation exchange,

- ion-exchange desalting;

- desalting by reverse osmosis method;

- decarbonization and deaeration;

- complex treatment;

- correctional treatment for corrosion and sediment's prevention, and for hardness scaling;

- other methods and their various combinations.

Membrane technologies are basis of modern approaches for water treatment in boiler houses. These technologies allow excluding a number of above-mentioned operations and this leads to decrease in material expenditures for receiving water of required quality.

\section{Theory and experimental methods}

Modern technologies allow creating a wide range of membranes which have good mechanical, thermal and chemical properties. Membrane devices and installations are also various. They are getting better and better from year to year.

However, there is one problem when using membranes of any type. This problem is formation of deposit layer on membrane surface. This layer is slowing down or completely stops membrane operation.

A formed layer of deposits is a result of concentration polarization. Preliminary purification of solutions which is carried out before membrane separation leads to increase in hardware registration of a production cycle and consequently also leads to increase in prime cost of an output product.

There are ways for prevention or decrease in impact of concentration polarization process. These ways are generally connected with design features of devices. [3]

So research of various constructive decisions and processing methods of impact on membrane processes for the purpose of their intensification is up to date.

Flat membrane devices are widely used in water treatment processes. These devices are simple in maintenance. Their advantage is the possibility of replacement only of that 
module where membrane is damaged. Replacement of all membrane surface is necessary if membrane surface is damage in devices of other types.

Figure 1 shows membrane operation of flat membrane device.

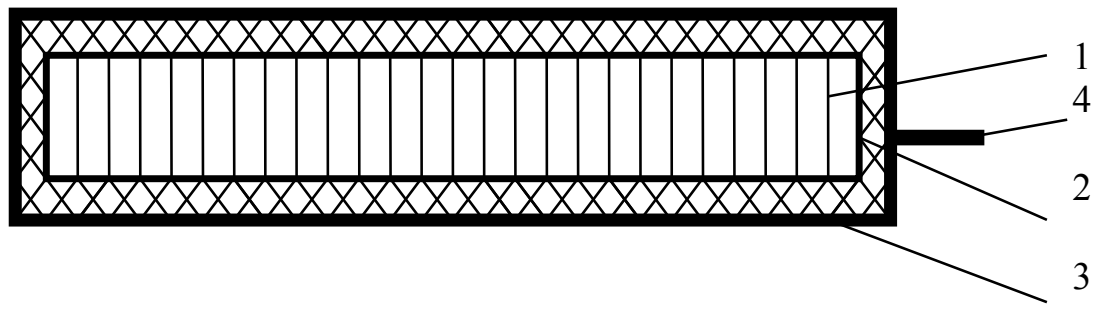

Fig. 1. Membrane element in cross section, where: 1-wire mash; 2-fabric substrate; 3-membrane; mounting bracket of an element.

Fabric substrate (2) is tensed on a mesh framework (1) of membrane element. Membrane (3) is above membrane substrates. It is put to an active layer of saturation solution.

Similar elements can have various positions in flat framework devices. They can be located in parallel order, in chessboard order and etc. Let's describe the mechanism of flat membrane element operation. Figure 2 shows the scheme of streams' movement which is formed when the device is operating

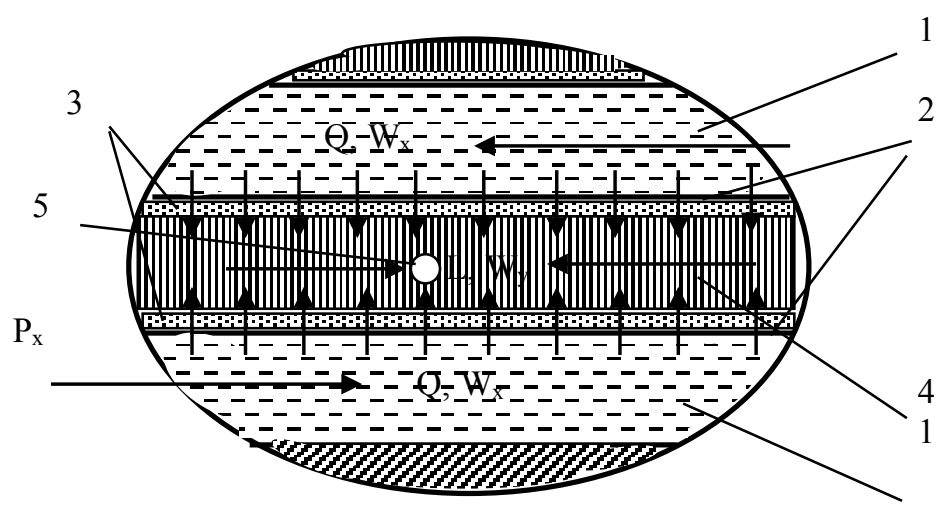

Fig. 2. Scheme of streams' movement in selected element of membrane device, where: 1 intermembrane channel; 2-membrane; 3 - fabric substrate; 4 - frame from wire mesh; 5 - orifice for permeate transferring.

Purified water stream moves in above membrane space (1). It moves with Wx speed in the mode of ideal replacement. Purified water streams come to submembrane space (4) through the top and low membranes (2). Streams mix up and with Wy speed a general stream moves to an orifice (5). And it is taken away from the device through this orifice.

Mathematical model specification of two-component saturation solution is described as multiphase continuous mixture. Saturation solution consists of liquid phase and mechanical inclusions. These inclusions are granules of various sizes. This is a mixture with variable concentration. A segment of intermembrane channel is studied in flat $0 \mathrm{X} 1 \mathrm{X} 2$ coordinate system. 
The mix having concentration $\mathrm{x} 1$ moves to the allocated area through A1B1 and C1D1 and comes out through $\mathrm{AB}$ and $\mathrm{CD}$ with $\mathrm{x} 2$ concentration. Liquid component of the solution partially passes through semi permeable membrane BB1 and CC1. And it is removed on permeable line $\mathrm{BC}$ if membrane thickness is $\delta \rightarrow 0$. As pore size of $\mathrm{d}$ membrane is much less than the sizes of mechanical inclusions there is deposits' layer formation of a disperse phase under ultrafiltration. This interferes liquid phase passing through the membrane (fig. 3).

Differential equation system of multicomponent medium was used to simulate ultrafiltration problem of two-component solution [4]. The first two equations are respectively laws of momentum and mass conservation.

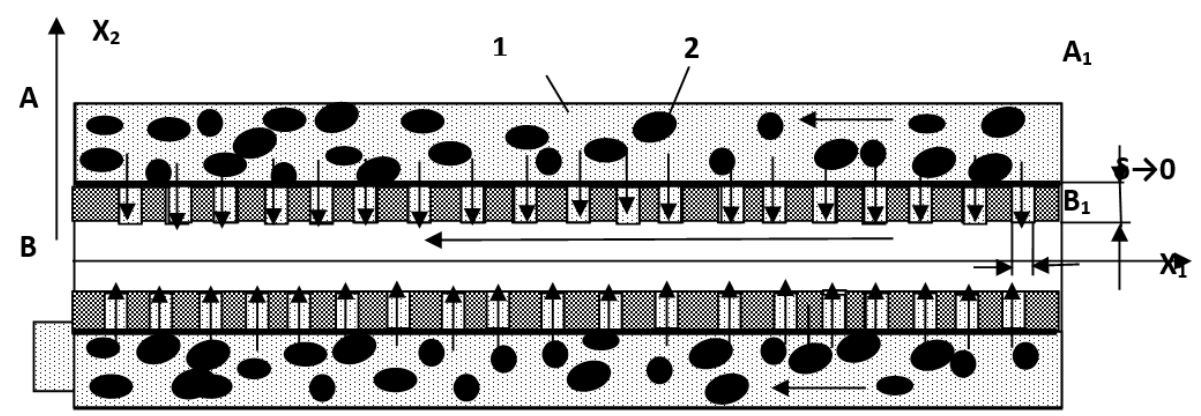

Fig. 3. Schematic representation of binary solution separation: 1 - liquid medium; 2 - mechanical inclusion.

The third equation is rheological. It states connection between stress tensor and strainrate tensor, average volume of solution concentration during the separation process and temperature mix.

$$
\left\{\begin{array}{l}
\frac{\partial \bar{V}_{L}}{\partial t}+\hat{V}_{L} \operatorname{div}\left(\bar{V}_{L}\right)=\frac{1}{\rho_{L}}\left(\nabla \bar{P}_{L}+\bar{F}_{n L}\right)+\bar{g} \\
\frac{\partial V_{L}}{\partial t}+\nabla\left(v_{L} \bar{V}_{L}\right)=0 \\
P_{L}=P_{L}\left(v_{L} ; D_{L} ; T\right)
\end{array}\right.
$$

where $\mathrm{V}_{\mathrm{L}}$ is velocity vector of two-component solution material, i.e.

$\mathrm{t}$ is residence time of the solution in the apparatus;

$\rho_{\mathrm{L}}$ is an average density of the solution in the separation process;

$\mathrm{F}_{\mathrm{nL}}$ is volume force of interelement interaction $\left(\bar{F}_{n L}=-\bar{F}_{L n}\right)$;

$\bar{g}$ is gravity acceleration;

$v_{\mathrm{L}}=\rho_{\mathrm{L}} / \rho_{\mathrm{mL}}$ is volume concentration of $\mathrm{L}$ material $(\mathrm{L}=1,2)$;

$\rho_{\mathrm{m} 1}$ is liquid density;

$\rho_{\mathrm{m} 2}$ is material density of dispersed phase particles;

$\mathrm{P}_{\mathrm{L}}$ is stress tensor;

$\mathrm{D}_{\mathrm{L}}$ is solution strain rate;

For a flat problem in the projection on $0 \mathrm{X}_{1} \mathrm{X}_{2}$ coordinate system axis, equations for the liquid phase will be as follows [13]: 


$$
\left\{\begin{array}{l}
\frac{\partial V_{1 L}}{\partial t}+V_{1 L} \frac{\partial V_{1 L}}{\partial X_{1}}+V_{2 L} \frac{\partial V_{1 L}}{\partial X_{2}}=\frac{1}{v \rho_{0}}\left(\frac{\partial P_{11 L}}{\partial X_{1}}+\frac{\partial P_{12 L}}{\partial X_{2}}\right) \\
\frac{\partial V_{2 L}}{\partial t}+V_{1 L} \frac{\partial V_{2 L}}{\partial X_{1}}+V_{2 L} \frac{\partial V_{2 L}}{\partial X_{2}}=\frac{1}{v \rho_{0}}\left(\frac{\partial P_{12 L}}{\partial X_{1}}+\frac{\partial P_{22 L}}{\partial X_{2}}\right) \\
\frac{\partial v_{L}}{\partial t}+\frac{\partial\left(v_{L} V_{1 L}\right)}{\partial X_{1}}+\frac{\partial\left(v_{L} V_{2 L}\right)}{\partial X_{2}}=0
\end{array}\right.
$$

For the second phase of solid inclusions on 0X1X2 coordinate system axis, these equations will be as follows:

$$
\left\{\begin{array}{l}
P_{122}=P_{0}+\alpha_{0}^{\prime}\left(\frac{\partial V_{12}}{\partial X_{2}}+\frac{\partial V_{22}}{\partial X_{1}}\right) \\
P_{222}=P_{0}+\alpha_{0}^{\prime}\left(\frac{\partial V_{12}}{\partial X_{1}}+\frac{\partial V_{22}}{\partial X_{22}}\right) \\
P_{122}=P_{0}+\alpha_{12}\left(\frac{\partial V_{12}}{\partial X_{2}}+\frac{\partial V_{22}}{\partial X_{1}}\right)
\end{array}\right.
$$

where stress tensor P can be as a sum of two stresses: equilibrium, depending only on the volume concentration and dissipative, depending on the volume concentration and strain rate

$$
\begin{gathered}
P=P_{0}(v)+P(v, D) ; \\
P_{0}(v)=\alpha_{0} I ; \\
\text { As } D \rightarrow 0, P \rightarrow P_{0}, \text { so } \\
-P_{0}=-\alpha_{0} \frac{v-v_{0}}{v_{0}-v}
\end{gathered}
$$

where $\alpha_{0}^{\prime}, \alpha_{1}$ are parameters determining dissipative properties of the dispersed phase.

Under ultrafiltration process productivity value (transfer of liquid mass phase through the filtering partition) of two-component solution segmentation is constantly decreased. This fact is stated by the results of numerical calculations on proposed model. 
Inflow ratio $\gamma$ is changed due to membrane pollution. Inflow ratio of the first material is inversely proportional to inflow ratio of the second material. Analyzing the data obtained during the experiment, we came to a conclusion that inflow ratio of a liquid phase of twocomponent solution depends on density of deposits' layer on a membrane. Inflow ratio decreases with the increase in thickness of deposits' layer.

Such mathematical transformations confirm surface increase of membranes in a device. This can be done by means of various layouts which use membrane elements in a module. It should be done for more efficiency of separation process.

Increase of membrane elements space, their form modification, use of various arrangement schemes assumes creation of an additional stream turbulization in the membrane device. This leads to unstable standing waves formation which can destroy deposits' layer.

\section{Experimental study}

An additional processing method is necessary for steady standing waves creation. This method allows keeping stationary mode of their operation for a long time without design change of membrane devices.

Such processing method is vibration impact on the separation solution. [5]

Introduction of resonance frequency vibrations into the separation solution allows decreasing negative impact of the formed deposits' layer on ultrafiltration process productivity.

Water flow moved to intermembrane channels of the device under excessive pressure. Pressure pulsations and mass-flow rate perturbation are introduced into separation solution by vibrating device. Pulsations have maximum amplitude and resonant frequency. These parameters are chosen experimentally on membrane productivity correlation on a permeata from vibrations' frequency.

Figure 4 shows the scheme of experimental installation. Electrodynamic vibration generator was installed to study influence of vibrations on ultrafiltration. The generator introduces separation vibration stream.

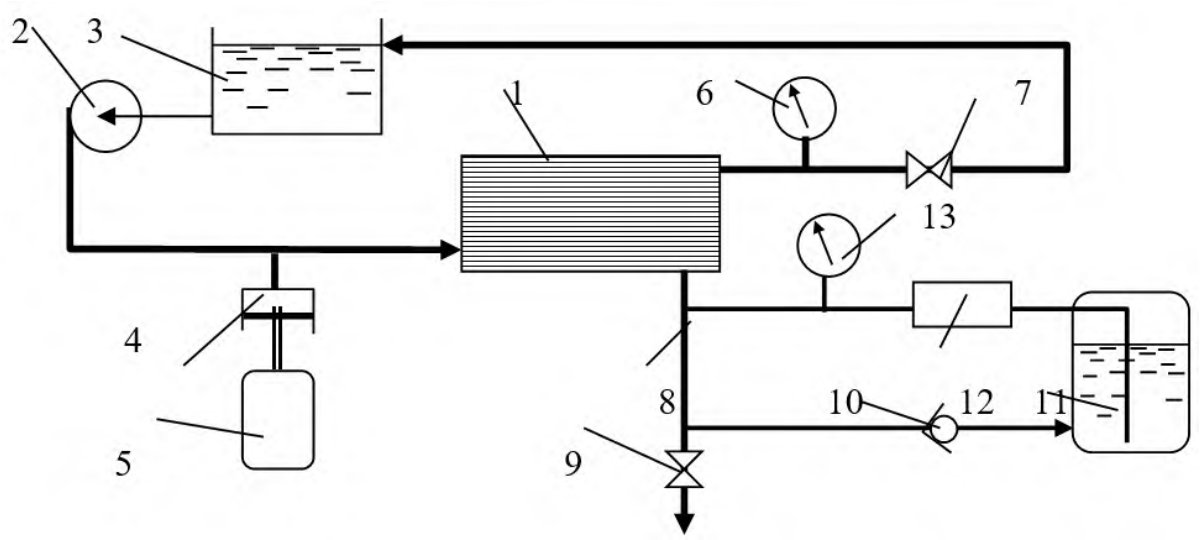

Fig. 4. Scheme of experimental installation with electrodynamic vibration generator, where: 1membrane device; 2 pump; 3-intermediate tank; 4-vibrotransferring knot; 5-vibration generator; 6, 13 - manometer; 7, 9-valve; 8- filtrate withdrawal line; 10-return valve; 11-hydropneumatic accumulator; 12-vibrating breaker. 
Power and control system provides control of key parameters defining reproduced vibration. In this case key parameters were amplitude and frequency changes of alternating current in moving coil of vibration generator 5 .

Electric fluctuations generator of sound frequency range was used for power supply of vibration generator. Applied frequency range was 5-5000 Hz. Vibration impact on circulating solution from electrodynamic generator was transmitted through a special vibrotransferring knot 4 . Rod vibration was done by means of sinusoidal or harmonious law. Rod vibration is mechanical oscillations of certain frequency and amplitude.

Fluctuations' intensity was determined by acceleration amplitude ratio to gravity acceleration

$$
\lambda=\mathrm{A} \omega^{2} / \mathrm{g}
$$

where $\omega$ is angular frequency ( $\mathrm{rad} / \mathrm{s})$. It can be expressed through an oscillation period $T$ (s) and oscillation frequency $f(\mathrm{~Hz})$.

\section{Results and discussions}

After mathematical modeling and experimental research it is possible to say that ultrafiltration process productivity increases by $30 \%$ in frequency range of $60-70 \mathrm{~Hz}$. And productivity peak is at $67 \mathrm{~Hz}$. (figure 5). [5]

According to these results we came to a conclusion that vibrations brought into a stream of purified water promote intensive turbulization increase of the stream which destroys a layer of formed deposits.

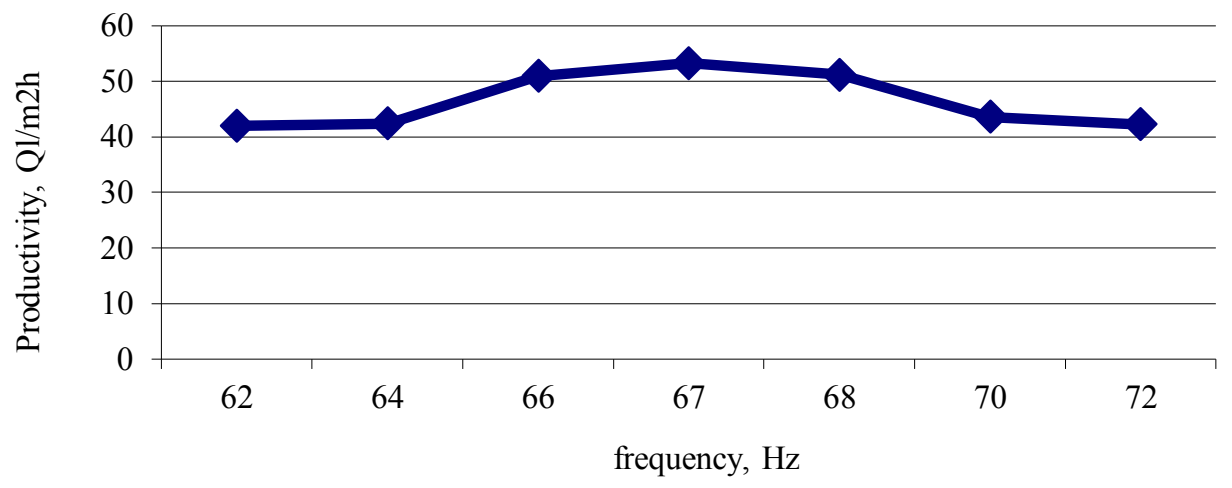

Fig. 5. Dependence of membrane specific productivity on vibrations' frequency.

Vibrations made by electrodynamic vibration generator in device's intermembrane channel (figure 4) are transferred directly into separation stream and produce speed V which changes according to harmonious law

$$
\mathrm{V}=\mathrm{V}_{0}+\Delta \mathrm{V} \sin \omega \mathrm{t}
$$

where $\Delta \mathrm{V}$ is oscillating speed amplitude.

It leads to standing wave formation which destroys undesirable deposits which are formed on the membrane surface.

\section{Conclusions}


The use of membrane technologies with vibration impact on cleaned water stream which passes through membrane device can increase operation of boiler houses. These technologies should be used in the system of water treatment in boiler houses using surface water for their operation. Saved funds for various reagents and processing equipment acquisition can increase economy of an enterprise operation and improvement of ecological indicators.

\section{References}

1. E.M. Kuvardina, S.S. Fedorov, Teorija i praktika sovremennoj nauki 12(18), 8 (2016)

2. S.I. Zheludkov, E.M. Kuvardina, Modernizacija kotel'noj, kak sposob povyshenija ee jeffektivnosti. Problemy $i$ perspektivy razvitija Rossii: Molodezhnyj vzgljad $v$ budushhee. Sbornik nauchnyh statej (ZAO Universitetskaja kniga, Kursk, 2018)

3. V.A. Kudrjavcev, V.V. Spichak, E.M. Kuvardina, Hranenie i pererabotka sel'hozsyr'ja 1, 44-47 (2001)

4. E.M. Kuvardina, Perspektivy membrannyh tehnologij ochistki mnogokomponentnyh rastvorov: monografija (Jugo-Zap. gos. Un-t., Kursk, 2012)

5. E.M. Kuvardina, O.G. Loktionova, K voprosu ob optimizacii parametrov vibracionnoj ultrafil'racii saharnyh rastvorov. Sbornik nauchnyh trudov "Vibracionnye mashiny $i$ tehnologii» (GTU, Kursk, 2005) 